\title{
ESTILO DE VIDA SALUDABLE EN TIEMPOS DE PANDEMIA
}

Healthy lifestyle in times of pandemic

\author{
Vilma Amparo Junchaya Yllescas ${ }^{3}$ \\ Universidad Roosevelt, Perú
}

Comer bien no basta para tener salud. Además, hay que hacer ejercicio, cuyos efectos también deben conocerse. La combinación de ambos factores constituye un régimen. Si hay alguna

deficiencia en la alimentación o en el ejercicio, el cuerpo enfermará". Hipócrates

\section{RESUMEN}

En el presente capítulo analizaremos las diversas variaciones en el estilo de vida a nivel familiar, debido a la influencia de la crisis sanitaria ocasionada por la pandemia del COVID-19 y el avance en el logro de los Objetivos del Desarrollo Sostenible, planteados en la

${ }^{3}$ Docente ordinario asociado, con grado de magister de la Universidad Peruana Cayetano Heredia, candidata al grado de doctor de la Universidad Enrique Guzmán y Valle, trabajando como docente en el sistema universitario por 21 años , ocupando diferentes cargos, natural de Ica, Química Farmacéutica de la Universidad San Luis Gonzaga de Ica, docente de la Universidad Franklin Roosevelt y la Universidad Peruana Los Andes, diplomada en "Atención Farmacéutica" de la Universidad Nacional Mayor de San Marcos, Diplomada en "Gestión y Gerencia de los servicios de Salud" de la Universidad Ricardo Palma, Diplomada en Farmacia Clínica de la Universidad Ricardo Palma; con estudios de Post- Grado en "Atención Farmacéutica" realizado en Buenos Aires Argentina y pasantía en ese país en "Control de Calidad" , "Fórmulas Magistrales" y "Atención Farmacéutica", también con pasantía en la Habana Cuba en el año 2007 en el Centro de Investigación del Desarrollo del medicamento. Correspondencia a ajunchaya@uroosevelt.edu.pe 
Agenda 2030, en especial el 3 que busca garantizar una vida sana y promover el bienestar de todos.

La pandemia de COVID-19 ha provocado cambios profundos en el comportamiento social, de tal forma que se debe tener en cuenta los efectos en la salud mental que pueden derivarse de estos cambios, también es necesario examinar posibles variaciones con respecto a los comportamientos y estilos de vida. Por ello los medios informativos han señalado cambios dramáticos en el sueño, consumo de sustancias, actividad física y dieta, que pueden tener consecuencias posteriores para la salud mental.

El COVID-19 ha provocado una crisis de salud pública sin precedentes en todo el mundo, que requiere varias acciones para minimizar la transición a una crisis social de larga duración, estudios proporcionarían evidencia necesaria para diseñar planes de prevención ante una posible nueva pandemia de trastornos psiquiátricos $\mathrm{y}$ cardiometabólicos comorbilidades.

Los Objetivos de Desarrollo Sostenible buscan una mejora en el bienestar, que debe reformularse y concretarse, porque se ha visto alterada a causa de la pandemia; la solidaridad mundial en tiempos de crisis es el mejor escudo frente a tan misteriosa enfermedad. También se hace un llamado a mayores esfuerzos de investigación para que aborden las consecuencias a corto y largo plazo de los cambios en los comportamientos de salud.

\section{INTRODUCCIÓN}

La salud es un derecho esencial y necesario para toda el mundo entero, que involucra un bienestar físico, psíquico y social, que implica una mejora constante de las condiciones personales y sociales en las que se desarrolla el individuo, con la finalidad de lograr un nivel de calidad de vida cada vez mejor, enfocada en la promoción, prevención e intervención de la salud desde una perspectiva multidisciplinaria y colectiva, ya sea a nivel comunitario, regional, 
nacional o internacional, es decir, no centrada en el individuo, sino en el colectivo (Miquel Bennassar, 2015).

Resulta complicado referirse a salud en tiempos de pandemia, más aún en Perú, cuando los índices de enfermedad y mortalidad, llegaron a picos altos que lo ubicaban en la cúspide en comparación con otros países. Se cree que el brote de COVID-19 se originó en Wuhan, China en diciembre 2019, desde entonces, el virus se ha propagado rápidamente, que ahora afecta a más de 200 países y territorios de todo el mundo. La Organización Mundial de la Salud (ONU, 2001), declaró pandemia mundial y una serie de medidas se han implementado con el fin de minimizar la propagación del virus. Algunos de estos incluyen distanciamiento social, autoaislamiento y cuarentena de aquellos que han contraído o potencialmente pudieran haber contraído COVID-19. Debido a la relativa rareza de las pandemias, la investigación sobre estos enfoques es escasa, pero está creciendo rápidamente. De hecho, investigaciones recientes han girado en un enfoque dirigido a las necesidades de salud mental de los trabajadores sanitarios de primera línea, otros también han enfatizado cómo implementar sistemas de apoyo psicológico durante la pandemia para asegurar estabilidad emocional. Actualmente se están realizando intentos para comprender la naturaleza de las consecuencias psicológicas derivadas de estas medidas y los esfuerzos iniciales de investigación están vinculados principalmente a la salud mental que los individuos pueden experimentar como resultado del aislamiento y cuarentena.

Mientras que tal enfoque es comprensible, también es necesario identificar cambios relacionados en los comportamientos de salud que puede estar ocurriendo a nivel de población para comprender mejor el rango de consecuencias psicosociales del reciente brote y sus medidas de contención asociadas. De hecho, con grandes segmentos de la población en condiciones de aislamiento, modificaciones a los comportamientos de estilo de vida son en gran 
parte inevitables. Es probable que estos incluyan cambios en el sueño, consumo de alcohol, actividad física, así como hábitos alimentarios e incluso la incidencia de casos de violencia (Bellomio C; García S, 2020). La investigación con fenómenos parecidos, no puede trazar paralelos entre pandemias, no respecto a los efectos del COVID-19, ni las medidas sociales restrictivas, puesto que pandemias anteriores con algo de similitud solo tenemos la del SARS en 2003. Si bien una importante proporción de individuos en cuarentena durante esta pandemia informó estar angustiado, como se evidencia por la proporción que mostró síntomas de depresión, este estudio busca profundizar los impactos en el estilo de vida o comportamientos de salud, debido a la pandemia de 2020. Se abordará la conceptualización de estilo de vida saludable en sus cuatro aristas principales: Actividad física y ejercicio, dieta y sueño.

\section{ESTILO DE VIDA SALUDABLE}

La soledad y el aislamiento social están asociados con mala salud física y mental y puede aumentar la probabilidad de trastornos mentales comunes (depresión y trastornos de ansiedad) (Sudriá et al., 2020). Durante largo tiempo la gente alrededor del mundo se ha aislado, absteniéndose a la interacción social debido a la pandemia COVID-19. Es necesidad imperiosa de la salud pública y la atención preventiva, proporcionar a los individuos, comunidades y agencias de salud, información e intervenciones para mantener el estilo de vida lo más saludable posible mientras se está aislado.

Los comportamientos de estilo de vida saludable consistentemente se han asociado con una reducción de la mortalidad por todas las causas, y aumento de la vida útil y el bienestar. Comportamientos poco saludables (dieta de mala calidad, falta de ejercicio físico, consumo de tabaco y alcohol) son principales contribuyentes a la carga mundial de morbilidad y también se han asociado con peores resultados en los trastornos psiquiátricos (Ossa Ramírez et al., 2005). Además, se reconoce que los estilos de vida 
poco saludables pueden ser una fuerza impulsora en la epidemia de trastornos mentales comunes. Las evidencias sugieren que el autoaislamiento obligatorio relacionado con la pandemia puede desencadenar depresión y postraumático trastorno de estrés y que, al ser un trabajador de la salud o tener COVID-19 son factores de riesgo para los trastornos psiquiátricos relacionados con el estrés. Dada la falta de tratamientos efectivos para COVID-19, intervenciones no farmacológicas son obligatorios para disminuir la transmisión de enfermedades.

La "nueva normalidad" incluye restricciones personales, físicas, políticas y un distanciamiento, como el confinamiento masivo y aislamiento domiciliario obligatorio. Estas pueden modificar, para bien o para mal, comportamientos de estilo de vida. Aumentado costumbres como una alimentación poco saludable y comportamiento sedentario, y disminución del tiempo al aire libre. Estos comportamientos pueden tener consecuencias imprevistas a medio y largo plazo para la salud física y mental. Por ejemplo, la disminución de la actividad física puede ampliar la gama de efectos negativos como los cardio-metabólicos y mentales. La revisión bibliográfica se ha centrado principalmente en los impactos de aspectos psicológicos, comportamientos de estilo de vida incluyendo cambios en la dieta, actividad física restringida, entre otros. El COVID-19 ha provocado una crisis de salud pública sin precedentes en todo el mundo. Desde mi perspectiva, se requieren varias acciones para minimizar la transición a una crisis social de larga duración.

Es hora de haya intervenciones que comiencen a incluir pautas de estilo de vida con el objetivo de traducir la evidencia en políticas de salud pública. Esto es crucial para los grupos vulnerables, como los de bajo ingreso familia, niños, ancianos, individuos socialmente aislados y personas con graves trastornos mentales. En cuanto a las pautas de estilo de vida, revisiones recientes han enfatizado el papel de la salud en mantener el estado nutricional y realizar actividades 
físicas (ejercicio en casa). Recomendaciones similares se realizaron en el momento de la pandemia de influenza en 1918, cuando las enfermeras de salud pública se adhirieron a los preceptos de buena higiene, nutrición, aire puro y descanso (Añez et al., 2006). Sin embargo, estas pautas de estilo de vida no son totalmente basadas en evidencia. De hecho, son básicamente la misma guía que se utiliza durante las situaciones no pandémicas veces. Datos de observación sobre cómo el público en general se ocupa en realidad del cuidado personal, la nutrición, la actividad física o falta de sueño reparador durante el confinamiento, representan una brecha de investigación. Para abordar esa brecha, los estudios observacionales del estilo de vida y comportamientos durante el aislamiento obligatorio son oportunos y claramente un paso necesario para el diseño de políticas públicas racionales y efectivas. Tales estudios proporcionarían esa evidencia tan necesaria para diseñar intervenciones para prevenir una nueva pandemia de trastornos psiquiátricos y cardiometabólicos comorbilidades.

Además, la recopilación de datos debe ser rápida y brindar información útil y confiable a autoridades sanitarias, medios de comunicación y ciudadanos. La psiquiatría y la medicina del comportamiento pueden ser particularmente beneficiado de encuestas e intervenciones llevado a cabo de forma remota para llegar a un gran número de personas necesitadas. Las encuestas a gran escala requieren redes internacionales para abordar cambios en los comportamientos de estilo de vida a consecuencias del COVID-19, este es un trabajo conjunto y multidisciplinar, donde se adopta tecnologías de información y comunicación como los dispositivos móviles, encuestas de seguimiento en línea y tecnologías de big data, recolección de datos remoto utilizando las redes sociales, la georreferenciación y las herramientas disponibles proporcionadas por la ciencia de datos. Tales herramientas proporcionan información de 
distintos grupos sociales en tiempo real, datos necesarios para informar a los responsables políticos.

La Organización Mundial de la Salud define los estilos de vida saludables como una "Forma general de vida basada en la interacción entre las condiciones de vida en un sentido amplio y los patrones individuales de conducta determinados por factores socioculturales y características personales", según ellos en el Perú, más del 50\% de la carga de enfermedades está asociadas a las Enfermedades No transmisibles (ENT), las cuales afectan a todos los grupos de edad y representan un grupo heterogéneo de dolencias, las cuatro principales son: enfermedades cardiovasculares, cáncer, diabetes y enfermedades respiratorias crónicas, estas enfermedades se desarrollan por un conjunto de factores de riesgo, prevenibles la mayoría de ellos. Destacan: el consumo de tabaco, uso nocivo del alcohol, régimen alimentario poco saludable y baja actividad física. Muchas de estas enfermedades serían evitables si la población adquiriera hábitos de vida saludables, entre ellos: realizar actividad física al menos 150 minutos semanales, consumir diariamente cinco porciones entre frutas y verduras, reducir al mínimo la ingesta de sal, alcohol y eliminar el consumo de cigarrillo (Cerón Sousa, 2015).

En consonancia con los principios de promoción de la salud, se parte de la premisa que la salud se crea y se vive en el marco de la vida cotidiana, siendo importante los centros de enseñanza desde la educación inicial, esta es una política de antaño en nuestro país y busca la sostenibilidad de la nación.

El entorno saludable es el espacio físico, social y cultural donde se habita cotidianamente (vivienda, escuela, lugar de trabajo, vecindario, vereda, municipio, ciudad) y donde se establecen relaciones sociales que determinan una manera de vivir y de ser, el concepto de Entornos Saludables incorpora tanto los aspectos de saneamiento básico, como los relacionados con espacios físicos limpios y adecuados, así como las redes de apoyo para lograr ámbitos 
psicosociales sanos y seguros, libres de violencia (abuso físico, verbal y emocional). De la misma manera las actividades de información y de educación para la salud que constituyen un complemento de estas otras formas de intervención. Por lo anteriormente dicho es importante destacar también que el buen estilo de vida saludable, es aquel en el que se mantiene una armonía y equilibrio en su dieta alimentaria, actividad o ejercicio físico, vida sexual sana, conducción segura, manejo del estrés, capacidad intelectual, recreación (sobre todo al aire libre) descanso, higiene, paz espiritual buenas relaciones interpersonales, así como también la relación con nuestro entorno entre otras.

Para llevar una vida saludable deben evitarse todo tipo de excesos; es tan malo comer mucho como comer muy poco, en este sentido deben escogerse los alimentos más nutritivos, en especial cereales, frutas, verduras, y beber abundante agua (dos litros de agua o más al día), para mantener un peso corporal adecuado (Lorenzo Berajona, Jose; Salvador Arriaga, Edgar; Rodríguez Bejarano, Sebastian; Aguilar Claros, 2016).

\section{ACTIVIDAD FÍSICA Y EJERCICIO}

Los comportamientos están fuertemente entrelazados con salud mental y se ha visto afectados a raíz de las medidas antipandémicas, la actividad física y el ejercicio moderado, son igual de importantes para tener buena salud.

La actividad física por sí sola se ha identificado como un factor protector importante en la reducción del riesgo de desarrollar depresión. Además, los aspectos sociales del comportamiento se interpretan cada vez más como un factor protector, similar a los comportamientos de estilo de vida identificado anteriormente. En un estudio a gran escala, fue informó que la actividad física, el consumo de alcohol, tabaquismo, índice de masa corporal y regularidad de interacción social estaban todos asociados con resultados específicos de salud mental (Posada, 2013). De hecho, existe una creciente 
evidencia de que las malas relaciones sociales tienen un efecto adverso sobre el funcionamiento de la salud.

Los efectos beneficiosos de la actividad física se regulan en muchos resultados de salud óptima. Además, se ha identificado consistentemente una gama de beneficios específicos tales como mejoras físicas, salud mental y bienestar. Por ejemplo, las revisiones sistemáticas apuntan al papel positivo de la relación entre el ejercicio regular y la calidad de sueño.

Desafortunadamente, las restricciones que rodean interacción social y actividades al aire libre, por motivo de la pandemia, resultan interrumpidas, por la paralización de las actividades diarias de millones de personas. Sin embargo, la importancia de la actividad física durante el bloqueo se ha enfatizado recientemente, argumentando que el ejercicio puede ayudar reequilibrar la salud física y mental y bienestar. Se concluyó que el ejercicio debe promoverse tanto como el distanciamiento social medidas durante estos tiempos difíciles (Sempere, Joaquim; Acosta, Alberto; Abdallah, Saamah; Ortí, 2010).

Varios estudios longitudinales han documentado las asociaciones entre aislamiento social y riesgo de mortalidad, así como desarrollo de enfermedades crónicas importantes, independientemente de otros factores sociodemográficos y condiciones de salud preexistentes. Una respuesta entre la relación de los malos comportamientos y el riesgo de la salud, con individuos socialmente aislados y solitarios, resulta en tener estilos de vida menos favorables, como el ausencia de actividad física (Lizandra \& Peiró-velert, 2020). Es inevitable que, para un gran número de personas, puestas en cuarentena, la ausencia de actividad física, realizada en los gimnasios, parque o instalaciones recreativas cerradas, por ejemplo, tenga un impacto negativo dramático. Sin embargo, muchas personas también pueden mantener, o incluso aumentar, su nivel de actividad física en este tiempo que probablemente sirva como factor protector contra la 
aparición de síntomas delicados en la salud mental. Como tal, una línea de investigación que puede ser beneficioso implicaría un examen de la actividad física y su relación con la salud mental en este contexto.

El sedentarismo, o cantidad de actividad física insuficiente para estar sano, es un gran problema de salud pública en el mundo y afecta a la mayoría (Bellomio C; García S, 2020). Es importante fortaleces los estilos de vida saludables, porque mejoran la conducta asumida como propias en nuestra vida cotidiana y que inciden positivamente en nuestro bienestar físico, mental y social. (Velázquez Cortés, 2009).

\section{DIETA Y ALIMENTACIÓN}

Un hábito principal es la alimentación, que va de la mano con el descanso y la práctica de la actividad física correctamente planificada. Por ello una alimentación saludable equilibrando su gasto y su consumo calórico, además de dar al cuerpo todo el descanso que necesita y sobre si en su estilo de vida incluye una práctica física continuada y sistemática; fortalece su salud (Ossa Ramírez et al., 2005).

Otro factor que indudablemente promueve la pobreza de hábitos alimentarios es el uso de dispositivos electrónicos y mayor exposición al tiempo tras de una pantalla, a través de los efectos del sedentarismo. Es probable que esto aumente con la educación en el hogar y restricciones de encierro, las campañas publicitarias de alimentos, snacks y comida poco saludable, aumenta los niveles de sedentarismo, trayendo como consecuencia el aumento de peso. En efecto, un grupo de investigación ya ha predicho el aumento de niveles de obesidad en infantes como como consecuencia directa de la pandemia de COVID-19 (Alvarez, Carlos Eduardo; Herrera Monge, María Fernanda; Herrera González, Emmanuel; Villalobos Víquez, Grettel; Araya Vargas, 2020). 
Por supuesto, la mala alimentación y el balance energético positivo no solo conducen al aumento de peso y la obesidad, sino también a un sinfín de enfermedades crónicas como, coronaria, tipo 2 diabetes mellitus, hiperlipidemia y otros. Dado que una mala alimentación favorece la aparición y exacerbación de múltiples enfermedades crónicas, es fundamental que los investigadores identifiquen primero cuales son las alteraciones en los patrones dietéticos durante la pandemia. Posteriormente, las consecuencias a largo plazo de estos hábitos dietéticos modificados durante el aislamiento social deben examinarse en todos los grupos de edad. Este tipo de agenda de investigación garantiza que las sociedades estén mejor equipadas con epidemias globales.

De las sustancias más vulnerables al abuso, el alcohol es abrumadoramente el más común en muchos países. Aunque el abuso de sustancias es técnicamente un trastorno de salud mental, el consumo de alcohol se identifica con frecuencia como un factor que contribuye a la decadencia física y subjetiva del bienestar. Medidas como la cuarentena y aislamiento social, a raíz de la pandemia de COVID-19, puede tener un efecto profundo en los niveles de consumo de alcohol en nuestro país.

Por ejemplo, en el norte de nuestro país se han extendido noticias sobre la compra abrumadora de cervezas. Tales aumentos pueden ser el sonido inicial de las campanas de alarma para el futuro, el consumo habitual y compulsivo puede convertirse en la norma para un número considerable de los individuos como respuesta al aburrimiento, la inactividad, aislamiento, y como medio para combatir la ansiedad, estrés y/o pérdida de sueño como resultado de las medidas introducidas para minimizar la propagación del virus. De hecho, hay llamados para mejorar la salud pública, advirtiendo sobre el consumo excesivo de alcohol durante el aislamiento, hay que evitar que este estilo de vida "de consumir alcohol" se normalice, evitando empujar a individuos ya vulnerables hacia patrones más peligrosos. 
El aislamiento social ya ha orillado a una gama de efectos negativos en el ámbito del uso de sustancias, individuos socialmente aislados tienen más probabilidades de abusar de las drogas, fuman cigarrillos, ser diagnosticados con trastorno por uso de sustancias y claro está el consumo excesivo de alcohol, en forma de embriaguez. El consumo de alcohol presenta múltiples efectos dañinos, por ejemplo, suprime el sistema inmunológico con efectos particulares sobre la capacidad pulmonar para combatir enfermedades infecciosas como COVID-19, un sistema inmunológico debilitado, por lo tanto, no sólo significa un mayor riesgo de infectarse con el coronavirus, sino también puede intensificarse gravedad y recuperación del virus; crece la violencia doméstica. La experiencia de la cuarentena parece estar vinculado a efectos negativos con respeto al alcohol, otra probabilidad futura serían los síntomas de dependencia y el crecimiento de pacientes con enfermedades respiratorias.

Los comportamientos dietéticos, pueden mejorar la ingesta energética, cuando estos son preparados de manera casera, puede ser un buen indicador ya que las comidas se cocinan utilizando elementos con menos conservantes o aditivos. Por otro lado, los comportamientos de comer bocadillos aumentan el estrés y otros efectos psicológicos.

\section{SUEÑO}

Los investigadores han afirmado que los individuos están durmiendo menos que hace décadas. Esta disminución observada en el sueño, respecto a la duración ha sido paralela al surgimiento de la vida enfermedades crónicas. Más recientemente, se ha afirmado que dormir es absolutamente fundamental para una salud óptima, ya que se encuentra evidenciado, que el sueño juega un papel fundamental en la aparición y exacerbación de casi todos los crónicos enfermedades (Ferré-Masó et al., 2020).

El aislamiento social y encierro, en esta línea del sueño, puede ser el lado positivo del COVID-19, ya que permite a la mayoría de las 
personas, disponer de más tiempo en casa permitiendo así, para algunos, flexibilidad en tiempos de sueño-vigilia y sueño prolongado duración.

Dado que Relación bidireccional entre el sueño y salud psicológica, la pérdida del sueño compromete el estado de salud mental. El general es probable que la población experimente más niveles normales de estrés. Por lo tanto, aquellos que están perdiendo el sueño, sufren alteraciones en los tiempos de sueño-vigilia y/o el aumento de los despertares nocturnos puede aumentar su riesgo a largo plazo de desarrollar enfermedades crónicas y es probable que también agrave los problemas de salud mental.

Las interacciones entre el sueño, la dieta y la actividad física están bien documentada, se sabe que los desequilibrios del sueño afectan la energía. homeostasis y es probable que el ciclo complejo de sueño inadecuado, promueva hábitos alimentarios más pobres, reduzca la motivación para hacer ejercicios, además, causa interrupciones a una variedad de hormonas metabólicas que están asociadas con obesidad y diabetes tipo 2 mellitus (Alvarez, Carlos Eduardo; Herrera Monge, María Fernanda; Herrera González, Emmanuel; Villalobos Víquez, Grettel; Araya Vargas, 2020). Por supuesto, estos comportamientos son impulsados por necesidades fisiológicas, pero estado psicológico y conexión social también juegan papeles importantes. Por ejemplo, comportamientos como comer, dormir y el ejercicio es más agradables cuando se realizan con otros y también se ven afectados por un estado psicológico del individuo. Los individuos que experimentan un sueño deficiente, son resultado de la ansiedad durante el encierro pueden intentar automedicarse con alcohol, creyendo que les ayudará a mejorar su sueño, pero desafortunadamente, esta no es una buena decisión.

El alcohol es un perturbador conocido del sueño y puede, en algunos casos, promueve la aparición del insomnio. Se sabe que la pérdida de sueño compromete función inmune, que es un factor 
fundamental perteneciente a COVID-19. Así, aquellos que están experimentando sueño más pobre durante el autoaislamiento necesitan primero reconocer y luego tomar acción correctiva de autoayuda para maximizar la inmunidad, protegerse contra la enfermedad del Coronavirus. El aislamiento social proporciona una ventana de oportunidad para desarrollar y mantener hábitos de sueño saludables y consistentes, pero solo si los individuos priorizan este comportamiento. En turno, esto puede ayudar a las personas a minimizar más adelante el desarrollo de enfermedades crónicas impulsadas por el estilo de vida, insomnio y o alcohol (u otras sustancias) de mal uso. Sería beneficioso realizar un seguimiento de la adopción tasas de acceso y suscripciones a la autoayuda programas de sueño correctivos en línea con las siguientes comparaciones en torno a la incidencia de fisiología y condiciones psicológicas en la fase prospectiva del COVID-19.

Para terminar con una nota más optimista, quiénes tienen más probabilidades de beneficiarse del bloqueo con respecto al sueño, son adolescentes, ellos suelen tener preferencias por dormir, lo más que puedan, cuando se le permita hacerlo, es por ello importante implantar en los centros educativos programas de rutina de sueño, que coadyuven a una mejora académica futura.

La investigación existente, sin embargo, indica que un mayor aislamiento social está asociado con tasas más altas de conductas sedentarias, incluso cuando edad, sexo, condiciones de vida, situación laboral, índice de masa corporal, antecedentes educativos, estado civil y salud general autoinformada están controlados (Tully et al., 2019). Por supuesto, El aislamiento social puede no corresponder directamente con el autoaislamiento.

Pero la investigación, como la anterior, da motivo de preocupación. Si bien varios proyectos están en curso para cuantificar los efectos en la salud mental de cuarentena y exposición al riesgo de 
contagio factores en todo el mundo, también hay una relación necesidad de identificar los factores que afectan los niveles de actividad física durante el aislamiento social, qué Los efectos a corto y largo plazo de estos pueden ser, junto con sus interacciones con la salud mental.

\section{BIENESTAR Y ESTILO DE VIDA SALUDABLE}

Es considerado derecho fundamental el libre desarrollo y bienestar de las personas, así como, la protección de su salud, la del medio familiar y la de la comunidad (MINJUS, 1993); de acuerdo a la cumbre de alto nivel sobre cobertura sanitaria universal organizada por las Naciones Unidas (ONU) y tomando en consideración la agenda 2030, fue considerado en el plan de acción para las personas, el planeta, la prosperidad, la paz y el trabajo conjunto, con la finalidad de erradicar con la pobreza de aquí al 2030 y promover una situación de riqueza y bienestar compartida, así como un desarrollo social y la protección ambiental de todos los países en el mundo.

Uno de los principales desafíos discutidos fue la necesidad de desarrollar un marco de referencia global con pautas clave para asegurar el acceso igualitario a los servicios de salud y lograr una mejor cobertura universal en enfermedades transmisibles y no transmisibles (Arredondo, Armando; Recamán, Ana Lucía; Castrejón, 1997). En el mundo diversos países enfrentan reto cobertura sanitaria universal con la finalidad de asegurar que todas las personas reciban los servicios sanitarios que necesitan, sin tener que pasar penurias financieras para pagarlos, y que lamentablemente difícilmente pueden resolverse para alcanzar el propósito; siendo uno de los mayores desafíos alcanzar una cobertura universal, que pueda ser capaz de asegurar que todas las personas reciban los servicios sanitarios que necesitan, sin tener que pasar penurias financieras para pagarlos (Boerma, Ties ; Evans, David ; Paule Kieny, Marie; Eozenou, Patrick; Evans, Tim; Wagstaf, 2014). 
Por tanto es necesario enfrentar y monitorear las resistencias de los sistemas de salud, ya sea las geográficas, financieras y resistencias cultural-organizacionales, mediante un sistema de salud sólido, eficiente y en buen funcionamiento, que satisfaga las necesidades de salud prioritarias en el marco de una atención centrada en las personas y que disminuya el efecto de 3 categorías de resistencia como problema de salud, ya sea de acceso, de poder y el acceso de los usuarios a los servicios de salud se incrementará significativamente (OPS/OMS Mexico, 2013).

¿Qué significa, entonces, bienestar? Un estudio reciente elaborado por el economista Paul Dolan y otros investigadores identificó cinco interpretaciones de bienestar en la literatura académica (Dolan et al., 2006). Una de ellas está estrechamente asociada a los ingresos; a mayor ingreso económico más recurso para el consumo, esto es asociado a la felicidad, es consumismo en exceso, hace a los individuos irracionales económicos. El segundo enfoque identifica el bienestar con una serie de "necesidades" objetivas, como salud, educación, ingresos, libertad política, etc. Las tres perspectivas restantes se centran en interpretaciones subjetivas del bienestar, y quizás las más conocidas sean la hedonista y la evaluativa. El enfoque hedonista identifica el bienestar con un equilibrio afectivo positivo, es decir, una ratio relativamente positiva entre emociones agradables y desagradables, estados de ánimo y sentimientos (Sempere, Joaquim; Acosta, Alberto; Abdallah, Saamah; Ortí, 2010).

La familia, como primer agente socializador, ejerce funciones que van más allá de la mera transmisión intencional y explícita de normas y valores, ya que proyecta una influencia crítica sobre el desarrollo social de los hijos, especialmente en aspectos como la competencia y la conducta pro social, cumple una función de apoyo, propiciando a los hijos el bienestar y confianza adecuados para desarrollar competencias y articular las relaciones con los otros, otorga al niño claves para construcción de representaciones globales acerca 
del funcionamiento de las interacciones sociales (Ramón Llopis, 2008).

La OMS en cierta medida se estaría librando de la responsabilidad de clarificar quién decide lo que es bienestar y salud, soslayando la controversia política que dichas definiciones acarrean, al mismo tiempo que deja entrever que la salud es un concepto científico "que se aplica a todos los grupos sociales y a todos los períodos históricos por igual" (Navarro, 1998). Dentro del aporte podemos mencionar que le bienestar se interrelaciona en tres dimensiones, física, mental y social. Existe una necesidad de invertir en salud y bienestar frente a los múltiples desafíos y adversidades, pudiéndose generar un "presupuesto de bienestar".

\section{PROMOCIÓN Y EDUCACIÓN PARA LA SALUD}

La promoción de la salud es el proceso que permite a las personas incrementar el control sobre su salud para mejorarla o lograr un autocuidado, que involucra acciones para incrementar habilidades y capacidades de las personas, sino también para persuadir en los cambios de condiciones sociales, ambientales y económicas que tienen impacto en los determinantes de salud (Ministerio de la Sanidad y el Consumo, 2003).

De hecho el empoderamiento de las personas, las familias, comunidades, será imprescindible para una respuesta eficaz a los problema de salud más relacionales y de origen social, tomando en cuenta que estas actitudes mejoran la alfabetización sanitaria que incluye un mayor conocimiento de la población y el desarrollo de habilidades personales que conduzcan a buen logro de la salud, además este proceso educativo deben responsabilizar a los ciudadanos en la defensa de la salud propia y colectiva y siendo una función importante de los profesionales sanitarios, sociales y de la educación su participación en todo momento (Ministerio de la Sanidad y el Consumo, 2003). 


\section{CONCLUSIONES}

Es importante para el ser humano adoptar estilos de vida saludable, respecto a la actividad física y ejercicio; dieta y alimentación; y sueño, son estos los factores del cuidado de la salud que conllevan al bienestar.

La mayor parte de la investigación psicológica sobre la pandemia de COVID-19 ha estado en los resultados de salud física, más que mental. Es fundamental producir una mejor comprensión del comportamiento, respecto a las alteraciones de la población en general y su impacto en la salud fisiológica y mental, que probablemente sean en su mayoría negativos. Se necesitan políticas, medidas y medios de salud para promover una mayor autoconciencia, autoayuda $\mathrm{y}$ autocuidado en el hogar para prevenir tensiones posteriores en el sistema sanitario.

La investigación sistemática sobre comportamientos relacionados con la salud, similar al de la salud mental, es necesario para comprender mejor el corto y largo plazo resultados de la crisis actual que afectan a muchas personas.

El COVID-19 ha provocado una crisis de salud pública sin precedentes en todo el mundo, que requiere varias acciones para minimizar la transición a una crisis social de larga duración, estudios proporcionarían evidencia necesaria para diseñar planes de prevención ante una posible nueva pandemia de trastornos psiquiátricos $\mathrm{y}$ cardiometabólicos comorbilidades.

Los Objetivos de Desarrollo Sostenible buscan una mejora en el bienestar, que debe reformularse y concretarse, porque se ha visto alterada a causa de la pandemia; la solidaridad mundial en tiempos de crisis es el mejor escudo frente a tan misteriosa enfermedad.

Para los profesionales de salud es importante que se involucren en educación para la salud creadas conscientemente 
destinadas a mejorar la alfabetización sanitaria que incluye la mejora del conocimiento de la población y el desarrollo de habilidades personales que conduzcan a la mejora de la salud.

\section{REFERENCIAS BIBLIOGRÁFICAS}

Alvarez, Carlos Eduardo; Herrera Monge, María Fernanda; Herrera González, Emmanuel; Villalobos Víquez, Grettel; Araya Vargas, G. (2020). Sobrepeso, obesidad, niveles de actividad física y autoestima de la niñez centroamericana: un análisis. Retos, 37(37), 85-92. https://recyt.fecyt.es/index.php/retos/article/view/71029/44611

Añez, G., Balza, R., Valero, N., Larreal, Y., \& De Citar, F. (2006). Impacto económico del dengue y del dengue hemorrágico en el Estado de Zulia, Venezuela, 1997-2003 Investigación original / Original research. Rev Panam Salud PublicaPan Am J Public Health Rev Panam Salud Publica, 1919(55), 314-320. https://doi.org/10.1590/S1020-49892006000500004

Arredondo, Armando; Recamán, Ana Lucía; Castrejón, B. (1997). Universal health coverage in the framework of the 2030 global agenda for sustainnable development: agreement and challenges. Nature, 388, 539-547.

https://www.ncbi.nlm.nih.gov/pmc/articles/PMC7100864/

Bellomio C; García S, G. R. G. S. J. M. J. L. (2020).

Recomendaciones para Mantener un Estilo de Vida Activo y Saludable en el. Sociedad Tucumana de Medicina y Deporte. https://radiotucuman.com/052020/59ce2e483cbbdb6fcc6c48439d8fc16d.pdf

Boerma, Ties ; Evans, David ; Paule Kieny, Marie; Eozenou, Patrick; Evans, Tim ; Wagstaf, A. (2014). Monitoreo del progreso hacia la cobertura universal de salud a nivel nacional y global Marco de trabajo , medidas y metas mayo de 2014. Grupo Del Banco Mundial, 14. https://apps.who.int/iris/bitstream/handle/10665/112827/WHO_ HIS_HIA_14.1_spa.pdf?sequence=1

Cerón Sousa, C. (2015). Universidad y Salud. UMH Sapiens 
Divulgación Científica, 2015(9), 28-29.

https://doi.org/10.21134/22553568.2015.9.us

Dolan, P., Peasgood, T., \& White, M. (2006). Review of research on the influences on personal well-being and application to policy making. DEFRA, London, August, 1-193.

Ferré-Masó, A., Rodriguez-Ulecia, I., \& García-Gurtubay, I. (2020).

Differential diagnosis of insomnia from other comorbid primary sleep disorders. Atencion Primaria, 52(5), 345-354. https://doi.org/10.1016/j.aprim.2019.11.011

Lizandra, J., \& Peiró-velert, C. (2020). Las relaciones sociales y su papel en la motivación hacia la práctica de actividad física en adolescentes: Un enfoque cualitativo. 2041, 41-47.

Lorenzo Berajona, Jose; Salvador Arriaga, Edgar; Rodríguez Bejarano, Sebastian; Aguilar Claros, A. (2016). Guía de entornos y estilos de vida saludables. Honduras, 1-83. http://iris.paho.org/xmlui/bitstream/handle/123456789/34580/vi dasaludable2016-spa.pdf?sequence $=1 \&$ is Allowed $=\mathrm{y}$

Ministerio de la Sanidad y el Consumo. (2003). FORMACIÓN EN PROMOCIÓN Y EDUCACIÓN PARA LA SALUD Informe. CONSEJO INTERTERRITORIAL DEL SISTEMA NACIONAL DE SALUD, 49(7), 1-12. https://doi.org/10.35362/rie4972047

MINJUS. (1993). Constitución Política del Perú. MINJUS. http://www.pcm.gob.pe/wpcontent/uploads/2013/09/Constitucion-Política-del-Peru1993.pdf

Miquel Bennassar, V. (2015). Estilos de vida y salud en estudiantes universitarios: La universidad como entorno promotor de la salud. [Universidad de Illes Balears]. In Universitat de les Illes Balears.

https://s3.amazonaws.com/academia.edu.documents/33091561/ tmbv1de1.pdf?AWSAccessKeyId=AKIAIWOWYYGZ2Y53U L3A\&Expires $=1526757748 \&$ Signature $=w G 3 g X d D 1$ JJkrOlUqs XzR6NwkFPs\%3D\&response-content-disposition=inline\%3B filename\%3DEstilos_de_vida_y_salud_en_estudiantes_ 
Navarro, V. (1998). La salud pública. Revista de Sanidad e Higiene Publica, 67(6), 417-418. https://ifdcsanluisslu.infd.edu.ar/sitio/material-de-estudio-del-ano2013/upload/navarro.pdf

ONU. (2001). Guía general para la aplicación de la Declaración del Milenio. Informe Del Secretario General, 52611, 69. http://www.aecid.es/CentroDocumentacion/Documentos/Informes y guías/Guxa_General_Aplicacion_Declaracion_Milenio_2001.p df

OPS/OMS Mexico. (2013). Covertura Universal el Salud OPS/OMS en México (OPS/OMS (ed.); Kunts Gráf). https://www.paho.org/mex/index.php?option=com_docman\&vi ew $=$ download \&alias $=855$-cobertura-universal-ensalud\&category_slug=ops-oms-mexico\&Itemid $=493$

Ossa Ramírez, J., González Velásquez, E., Rebelo Quirama, E., \& Pamplona González, J. (2005). Los conceptos de bienestar y satisfacción. Una revisión de tema. Revista Científica Guillermo de Ockham, 3(1), 27-59. https://doi.org/10.21500/22563202.472

Posada, J. A. (2013). La salud mental en Colombia. Biomédica, 33(4), 824. https://doi.org/10.1055/s-1981-29613

Ramón Llopis, G. (2008). Bienestar familiar y relaciones de amistad. Un estudio con adolescentes en el contexto escolar. IEEE International Symposium on Applications of Ferroelectrics, 3, 59-75. https://doi.org/10.1109/ISAF.2008.4693796

Sempere, Joaquim; Acosta, Alberto; Abdallah, Saamah; Ortí, M. (2010). Altered pathogenicity of avian myelocytomatosis (MC29) viruses with mutations in the v-myc gene. Virology, 124(1), 164-172. https://doi.org/10.1016/0042-6822(83)903008

Sudriá, M. E., Andreatta, M. M., \& Defagó, M. D. (2020). Los efectos de la cuarentena por coronavirus (COVID-19) en los hábitos alimentarios en Argentina. Diabeta, 38(171), 10-19. 
https://ri.conicet.gov.ar/bitstream/handle/11336/114882/CONI CET_Digital_Nro.72f7182e-88d0-49c5-8147 a7487391da3e_A.pdf?sequence $=2$

Velázquez Cortés, S. (2009). Hábitos y estilos de vida saludable. Programa Institucional Actividades De Educacion Para Una Vida Saludables, 3(1), 16. https://www.uaeh.edu.mx/docencia/VI_Lectura/licenciatura/do cumentos/LECT103.pdf 